\title{
Analysis of health-related quality of life in patients with brain tumors prior and subsequent to radiotherapy
}

\author{
CLAUDIA BITTERLICH and DIRK VORDERMARK \\ Department of Radiation Oncology, Martin Luther University Halle-Wittenberg, D-06120 Halle (Saale), Germany
}

Received February 1, 2017; Accepted March 31, 2017

DOI: $10.3892 / \mathrm{ol} .2017 .6310$

\begin{abstract}
Health-related quality of life (HRQOL) was evaluated for a patient cohort with benign or malignant brain tumors prior and subsequent to radiotherapy. The following inclusion criteria were applied: Sufficient compliance, understanding of patient information and the existence of a brain tumor without previous radiotherapy. Patients were asked to complete the European Organization for Research and Treatment of Cancer QLQ-C30 and QLQ-BN20 questionnaires at the following times: Prior to $(\mathrm{t} 0)$ and subsequent to $(\mathrm{t} 1)$ radiotherapy, and at $3(\mathrm{t} 2), 6(\mathrm{t} 3)$ and 12 (t4) months following treatment. In addition, at $\mathrm{t} 1$ the side effects were assessed according to the Common Terminology Criteria for Adverse Events. Generally, the global QOL, a standard term describing general QOL, improved slightly ( $\mathrm{t} 0=49 ; \mathrm{t} 4=65)$. At $\mathrm{t} 1$, a significant increase in fatigue, loss of appetite and alopecia was reported. During follow-up, the symptoms experienced by the patients decreased, and the global QOL remained constant. The objectively recorded side effects of the therapy were comparable with the patient-reported outcomes.
\end{abstract}

\section{Introduction}

Primary brain tumors are subdivided into benign and malignant types and classified according to the World Health Organization (WHO) Classification (1), ranging from e.g., WHO grade I meningioma to WHO grade IV glioblastoma. Symptoms vary according to the location of the tumor and include paralysis or sensory disturbances, intracranial pressure, personality changes, epileptic seizures and cognitive

Correspondence to: Professor Dirk Vordermark, Department of Radiation Oncology, Martin Luther University Halle-Wittenberg, Ernst-Grube-Strasse 40, D-06120 Halle (Saale), Germany

E-mail: dirk.vordermark@uk-halle.de

Abbreviations: HRQOL, health-related quality of life; GBM, glioblastoma multiforme; EORTC, European Organization for Research and Treatment of Cancer; CTCAE, Common Terminology Criteria for Adverse Events

Key words: quality of life, brain tumor, glioblastoma, meningioma impairments, are possible. All these symptoms may markedly affect health-related quality of life (HRQOL). HRQOL is a multidimensional construct that examines the subjective effects of diseases and therapy-associated symptoms on the well-being of patients. This includes physical, psychological and social functioning (2). During previous decades, HRQOL has become an important 'patient outcome' consideration in oncology when evaluating treatment results (3). In palliative cases, such as for patients with glioblastoma multiforme (GBM), the stabilization of HRQOL is the primary intention (4).

Understanding of the patterns of HRQOL is important for patient information and shared decision-making. Patients need to know what their HRQOL outcome will be during therapy, and also during palliative care. Individual problems may be identified with the assistance of QOL questionnaires prior to, during and subsequent to therapy; therefore, supportive care may be initiated (5). HRQOL surveys also tend to improve communication between the patients and physicians (6). For an effective evaluation of treatment outcomes, it is crucial to identify changes in HRQOL that occur as result of the tumor, the therapy or other issues (3). It has previously been demonstrated that the objective assessment of adverse events is often distinct from the subjective experience of the patient (7).

In order to evaluate the information currently available regarding $\mathrm{HRQOL}$, patients with primary brain tumors who were scheduled for radiotherapy were asked to complete QOL questionnaires prior and subsequent to their treatment series. Following radiotherapy, the objective adverse events were classified and subsequently compared with the subjective data obtained from the QOL questionnaires.

\section{Materials and methods}

Recruitment of patients. A total of 30 consecutive patients with primary brain tumors were enrolled, following written informed consent being obtained, between March 2009 and June 2010 at the Department of Radiation Oncology, Martin Luther University Halle-Wittenberg [Halle (Saale), Germany]. Patient characteristics and treatment details are presented in Table I. The patients were requested to complete the Core Questionnaire C30 and the module questionnaire BN20, from the European Organization for Research and Treatment of Cancer (EORTC). The study was approved by the Ethics Review Committee of the Medical Faculty, Martin Luther University Halle-Wittenberg. 
The following inclusion criteria were applied: Sufficient compliance, understanding of patient information describing the HRQOL study and the existence of a brain tumor without previously administered radiotherapy. Patients who had received prior irradiation or had insufficient cognitive skills to complete the HRQOL questionnaire, as assessed by the treating radiation oncologist, were excluded. The present study was longitudinal, consisting of five questionnaires completed during a 12-month period: Prior to ( $\mathrm{t} 0)$, at the end of radiotherapy (t1) and 3 months (t2), 6 months (t3) and 12 months (t4) following the end of radiotherapy. Questionnaires were distributed to patients at $\mathrm{t} 0$ and $\mathrm{t} 1$ in the Department of Radiation Oncology, Martin Luther University Halle-Wittenberg. At subsequent time points, the questionnaires were posted with a pre-stamped envelope. Other information regarding the patients and their therapy was extracted from medical records and anamnesis.

Questionnaires. For evaluation of QOL, the EORTC QLQ-C30 questionnaire version 3.0 (8) and the QLQ-BN20 questionnaire (9) were used. The BN20 was developed specifically for patients with primary brain tumors by the EORTC Quality of Life Group (9). The two questionnaires had previously been validated by the EORTC $(8,10)$. The QLQ-C30 consisted of 30 questions associated with functions and symptoms. Each question may be answered using 4 grades: 1, not at all; 2, low; 3 , moderate; and 4, high. There were two questions concerning global QOL scored from 1 (very poor) to 7 (excellent). According to the EORTC C30 scoring manual (11), the answers may be transformed into scales ranging from 0 to 100 . There are five functional scales (physical, role, cognitive, emotional and social functioning), nine symptom scales (fatigue, nausea and emesis, pain, dyspnea, sleep disturbance, loss of appetite, constipation, diarrhea and financial difficulties) and one scale for global QOL. The QLQ-BN20 consisted of 20 questions; the responses may be transformed into 11 symptom scales (future uncertainty, visual disorder, motor dysfunction, communication deficit, headaches, seizures, drowsiness, alopecia, itchy skin, weakness of legs and bladder control). For the functional scales and global QOL, high scores represent a good functioning and QOL. For the symptom scales, high scores indicate the presence of severe symptoms.

Common Terminology Criteria for Adverse Events (CTCAE). Adverse events were also objectively assessed at $\mathrm{t} 1$, using checklists based on the CTCAE (version 3) (12). The symptoms of alopecia, nausea, headache and fatigue were evaluated. According to CTCAE, symptoms are classified as grades 1-5: Grade 1, mild; grade 2, moderate; grade 3, severe; grade 4, life-threatening or disabling adverse event; and grade 5, mortality associated with adverse event. The symptom of alopecia was classified from 0 to 2 as an exception, as this toxicity cannot be severe or life-threatening. The objective adverse events assessed using the CTCAE checklists could be subsequently compared with the subjective responses from the QOL questionnaires.

Assessment and statistical analysis. The following five groups were formed: All patients $(n=30)$; malignant $(n=14)$; benign $(n=16)$; glioblastoma multiforme $(\mathrm{GBM} ; \mathrm{n}=10)$; meningioma
( $n=11)$. GMB and meningioma were the two largest subgroups (full patient characteristics are presented in Table I). Data at each time point were compared to t0 (prior to radiotherapy). The mean, median and standard deviation values were calculated. Statistical evaluation was performed using the non-parametric Mann-Whitney U test and STATISTICA version 10 software (StatSoft, Inc., Tulsa, OK, USA). Based on the multiple comparisons, $\mathrm{P}<0.01$ were considered to indicate a significant difference. Additionally, changes in clinical significance over time were considered, according to Osoba et al (13). This study defined which magnitude of change in HRQOL scores, assessed using EORTC questionnaires, corresponds to a change, noticed by the patient as significant. According to Osoba et al (13), a deviation in an individual score by 5-10 points indicated a slight change, by $10-20$ points indicated a moderate change, and by $>20$ points represented a high clinical relevance.

\section{Results}

Patient and treatment characteristics. The median age was 64.6 years (range, 25.9-80.4 years). A total of 14 patients exhibited a malignant brain tumor and 16 had a benign tumor. A total of nine patients also received chemotherapy (temozolomide) during and subsequent to radiotherapy. Detailed treatment characteristics are presented in Table I. During the 12-month follow-up period, eight patients succumbed: Of those, two patients succumbed after 3 months (t2), three patients after 6 months (t3) and additional three patients after the 12-month period ( $\mathrm{t} 4)$. The causes of mortalities were not analyzed; however, it was expected that in patients with malignant brain tumors, including glioblastoma, the mortalities were predominantly tumor-associated. All patient and treatment characteristics are summarized in Table I.

Questionnaire response rate. At t0 (prior to radiotherapy) and $\mathrm{t} 1$ (subsequent to radiotherapy), all questionnaires were completed $(100 \%$ response rate). In the whole group, the response rate among surviving patients was $78.6 \%$ at t2 (3 months), $81.5 \%$ at t 3 (6 months) and 63,6\% at t4 (12 months) which is predominantly $>70 \%$ response rate, the level which is typically aimed at achieving, and acceptable, considering the palliative situation among the malignant brain tumor patients. The poorest response rate was achieved after 12 months in the malignant group (50\%). At all time points, there were higher response rates from the benign and meningioma groups, compared with the malignant and GBM groups. The response rates for all surveys are presented in Table II. The results were generated from the proportion of questionnaires returned by patients who were alive each of the time points.

Changes to global QOL. There were no statistically significant changes recorded in global QOL for any of the subgroups observed. However, there was an increase of mean global QOL between $\mathrm{t} 0$ and $\mathrm{t} 4$, as indicated in Fig. 1, which was of a clinically significant extent, according to the definitions by Osoba et al (13) $(\mathrm{t} 0=49 ; \mathrm{t} 4=65)$. Similar results were identified in the following groups: Benign ( $\mathrm{t} 0=55 ; \mathrm{t} 4=66)$; meningioma $(\mathrm{t} 0=54 ; \mathrm{t} 4=72)$; malignant $(\mathrm{t} 0=42 ; \mathrm{t} 4=61) ; \mathrm{GBM}(\mathrm{t} 0=42 ; \mathrm{t} 4=67)$. Therefore, the results indicated that there was a moderate 
Table I. Baseline characteristics of all 30 patients.

\begin{tabular}{|c|c|}
\hline Characteristics & $\begin{array}{c}\text { No. of } \\
\text { patients }(\%)\end{array}$ \\
\hline Age, years [median (range)] & $64.8(25.9-80.4)$ \\
\hline \multicolumn{2}{|l|}{$\operatorname{Sex}$} \\
\hline Female & $16(53)$ \\
\hline Male & $14(47)$ \\
\hline \multicolumn{2}{|l|}{ Primary tumor } \\
\hline Glioblastoma & $10(33)$ \\
\hline Astrocytoma WHO III & $3(10)$ \\
\hline Oligodendroglioma & $1(3)$ \\
\hline Neurocytoma & $1(3)$ \\
\hline Craniopharyngioma & $1(3)$ \\
\hline Pituitary adenoma & $2(7)$ \\
\hline Meningioma & $11(37)$ \\
\hline Acoustic neuroma & $1(3)$ \\
\hline \multicolumn{2}{|l|}{ Tumor type } \\
\hline Benign & $16(53)$ \\
\hline Malignant & $14(47)$ \\
\hline \multicolumn{2}{|l|}{ WHO grade } \\
\hline I & $15(50)$ \\
\hline II & $2(7)$ \\
\hline III & $3(10)$ \\
\hline IV & $10(33)$ \\
\hline \multicolumn{2}{|l|}{ Chemotherapy } \\
\hline Yes & $9(30)$ \\
\hline No & $21(70)$ \\
\hline \multicolumn{2}{|l|}{ Irradiation } \\
\hline 3D-conformal & $15(50)$ \\
\hline Stereotactic & $15(50)$ \\
\hline $\begin{array}{l}\text { Planning target volume, } \mathrm{ml} \\
\text { [median (range)] }\end{array}$ & $106(9-476)$ \\
\hline Total dose, Gy [median (range)] & $54(45-60)$ \\
\hline $\begin{array}{l}\text { Duration of radiotherapy, days } \\
\text { [median (range)] }\end{array}$ & $42(14-50)$ \\
\hline $\begin{array}{l}\text { Karnofsky performance status prior } \\
\text { to radiotherapy, } \% \text { [median (range)] }\end{array}$ & $85(60-100)$ \\
\hline $\begin{array}{l}\text { Karnofsky performance status } \\
\text { subsequent to radiotherapy, } \% \\
\text { [median (range)] }\end{array}$ & $80(50-100)$ \\
\hline
\end{tabular}

WHO, World Health Organization.

increase in the mean global QOL for all participating groups. Compared with the groups receiving palliative therapy (malignant and GBM), the global QOL was higher in the groups receiving curative therapy (benign and meningioma) during duration of the present study (Fig. 2).

Subjective adverse events. At the end of radiotherapy (t1), patients experienced significantly higher mean levels of fatigue $(\mathrm{t} 0=29 ; \mathrm{t} 1=54 ; \mathrm{P}=0.002)$, appetite loss $(\mathrm{t} 0=11 ; \mathrm{t} 1=24 ; \mathrm{P}=0.008)$
Table II. Response rates to questionnaires among surviving patients at all time points from t0 (prior to radiotherapy) to $t 4$ (12 months).

Patients who responded at each time point $(\%)$

\begin{tabular}{lccccc}
\cline { 2 - 6 } Patient groups & $\mathrm{t} 0$ & $\mathrm{t} 1$ & $\mathrm{t} 2$ & $\mathrm{t} 3$ & $\mathrm{t} 4$ \\
\hline All patients & 100 & 100 & 78.6 & 81.5 & 63.6 \\
Benign & 100 & 100 & 81.3 & 87.5 & 68.8 \\
Malignant & 100 & 100 & 75.0 & 72.7 & 50.0 \\
Meningioma & 100 & 100 & 90.9 & 90.9 & 72.7 \\
GBM & 100 & 100 & 78.8 & 62.5 & 66.7
\end{tabular}

GBM, glioblastoma multiforme; t0, prior to radiotherapy; $\mathrm{t} 1$, following radiotherapy; $\mathrm{t} 2,3$ months after radiotherapy; $\mathrm{t} 3,6$ months after radiotherapy; t4, 12 months after radiotherapy.

\section{Global quality of life of all patients}

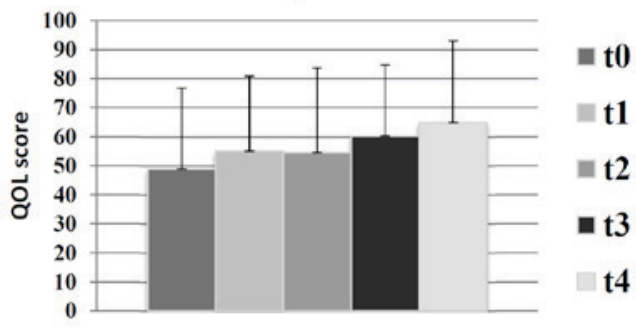

Figure 1. Global quality of life for all patients $(n=30)$ at all time points: t0 (prior to radiotherapy), t1 (subsequent to radiotherapy), t2 (after 3 months), t3 (after 6 months) and 4 (after 12 months), as measured by the European Organization for Research and Treatment of Cancer QLQ-C30 questionnaire, in which a higher score equated to an improved global quality of life.

and alopecia ( $\mathrm{t} 0=9 ; \mathrm{t} 1=31 ; \mathrm{P}=0.006)$ compared with the beginning of radiotherapy. At 3 months after radiotherapy (t2), there was a significant increase in reports of itchy skin $(\mathrm{t} 0=4 ; \mathrm{t} 2=26$; $\mathrm{P}=0.006)$. At the end of radiotherapy ( $\mathrm{t} 1)$, patients exhibited increased levels of itchy skin, however, this was not identified as significant $(\mathrm{t} 0=4 ; \mathrm{t} 1=19)$. Fig. 3 presents an overview of the surveyed symptoms scales for all patients.

Objective adverse events. The objective evaluation of the side effects using CTCAE following radiotherapy (t1) indicated certain types of therapy-associated side effects in $93.3 \%$ of the participants. The main issues that patients identified were fatigue and alopecia. In addition, $80 \%$ of patients demonstrated alopecia grade 1 (thinned or fragmentary). The results indicated that $90 \%$ of patients suffered from fatigue; of those, $56.7 \%$ had mild fatigue and $13.3 \%$ had severe fatigue. Overall, only two patients $(6.7 \%)$ were asymptomatic following radiotherapy at $\mathrm{t} 1$. There were specific differences between the subgroups. All patients in the GBM group exhibited alopecia grade 1 ; by contrast only $73 \%$ of patients with meningioma exhibited alopecia grade 1 and $27 \%$ reported no alopecia. There were higher grades of fatigue observed in the groups 


\section{Global quality of life}

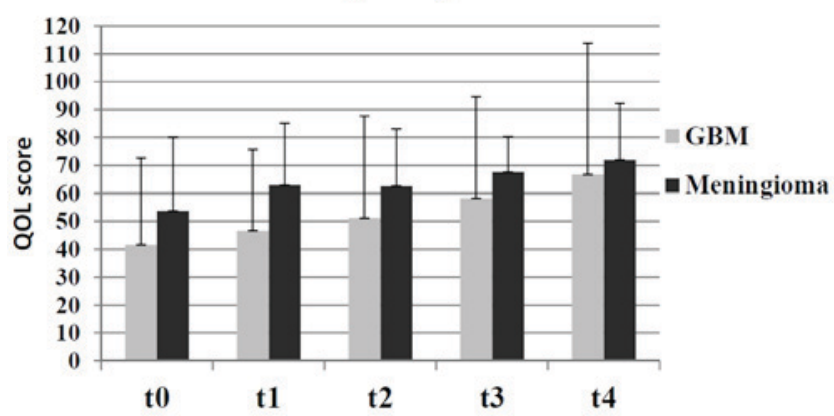

Figure 2. Comparison of global quality of life at all time points in the GBM (palliative therapy) and meningioma (curative therapy) groups, as measured by the European Organization for Research and Treatment of Cancer QLQ-C30 questionnaire, in which a higher score equated to an improved global quality of life. GBM, glioblastoma multiforme.

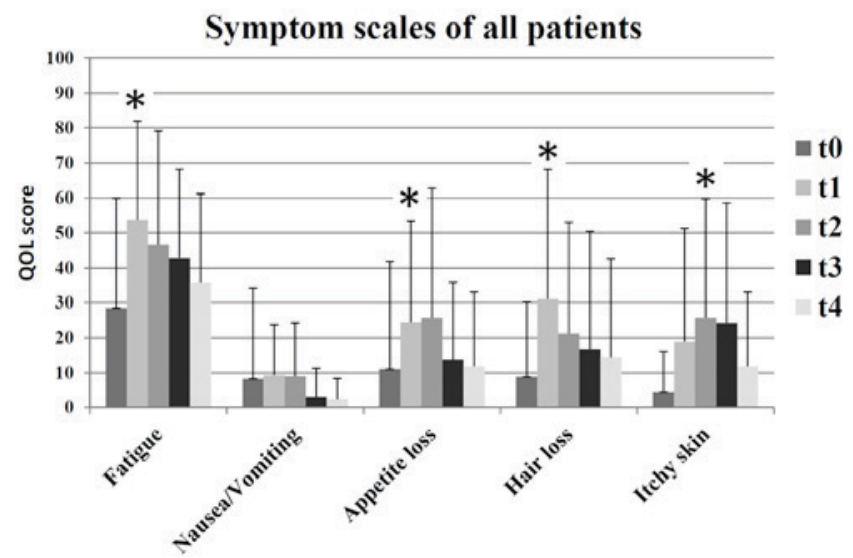

Figure 3. Symptom scales of all patients $(n=30)$ at all time points: $\mathrm{t} 0$ (prior to radiotherapy), t1 (subsequent radiotherapy), t2 (after 3 months), t3 (after 6 months) and t4 (after 12 months), as measured by the European Organization for Research and Treatment of Cancer questionnaire. A higher score indicated a worse quality of life. ${ }^{*} \mathrm{P}<0.01$.

with palliative therapy $(7.2 \%$ grade $0 ; 50 \%$ grade $1 ; 21.4 \%$ grade $2 ; 21.4 \%$ grade $3 ; 0 \%$ grade 4$)$ in comparison with the groups receiving curative therapy $(12.5 \%$ grade $0 ; 62.5 \%$ grade $1 ; 18.7 \%$ grade $2 ; 6.3 \%$ grade $3 ; 0 \%$ grade 4$)$. Only a small number of patients exhibited nausea $(23.3 \%)$ or headaches $(36,7 \%)$. The distribution of objective toxicity grades is presented in Fig. 4.

Differences between the subgroups. Patients with benign brain tumors demonstrated stable functional scales over time, revealing constant or improved values after radiotherapy in the five types of function measured using the EORTC QLQ-C30 questionnaire. However, in patients with malignant tumors, the functional scales became poorer from $\mathrm{t} 1$ (end of radiotherapy) and recovered slightly up until t 4 (after 12 months). The cognitive function decreased in the GBM group 12 months following radiotherapy $(\mathrm{t} 0=85 ; \mathrm{t} 4=58)$. Patients in the meningioma group exhibited more stable cognitive functioning ( $\mathrm{t} 0=88 ; \mathrm{t} 4=88$ ). There was an increase in future uncertainty in the GBM group after 12 months ( $\mathrm{t} 0=39 ; \mathrm{t} 4=58$ ), whereas future uncertainty continuously decreased in the meningioma group ( $\mathrm{t} 0=28$;
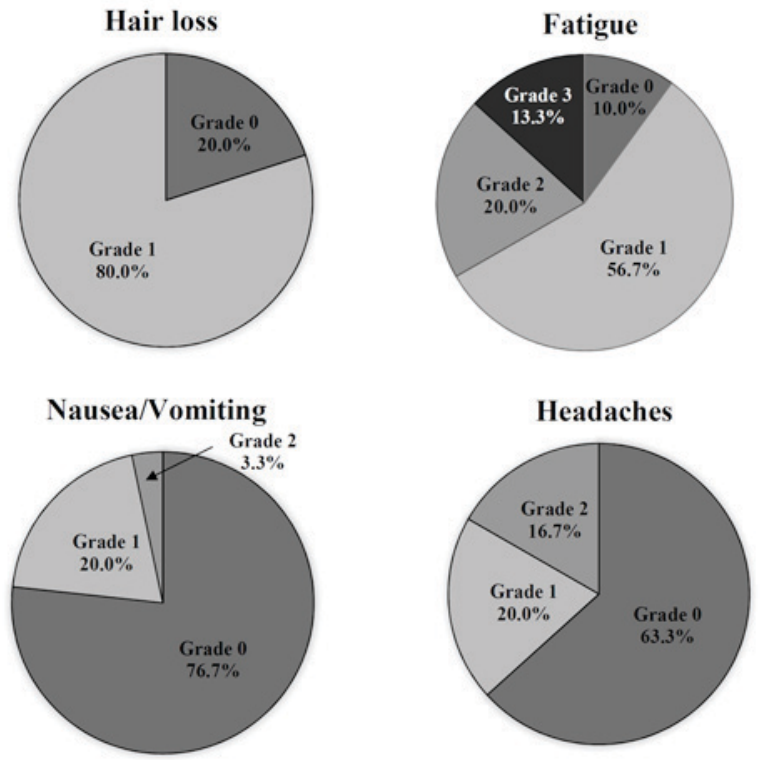

Figure 4. Objective adverse side effects experienced by patients $(n=30)$ at t1 (subsequent to radiotherapy), according to the Common Terminology Criteria for Adverse Events: Grade 0, no adverse side effect; grade 1, mild; grade 2, moderate; grade 3, severe; grade 4, life-threatening or disabling adverse side effect.

t4=17). Patients with meningioma experienced increased levels of alopecia during the study period, as compared with patients with GBM (Fig. 5).

An additional distinction identified was associated with financial difficulties (Fig. 6): Patients in the meningioma and benign groups exhibited less financial difficulties, compared with patients in the GBM and malignant groups. In addition, financial difficulties decreased at 4 (12 months following radiotherapy) in the groups receiving curative therapy, and markedly increased in groups with palliative therapy.

\section{Discussion}

The prospective evaluation of HRQOL in 30 patients with brain tumors revealed that, despite radiotherapy and associated side effects, there was no significant decrease in QOL observed in any subgroup. There was a moderate increase in global QOL 12 months following radiotherapy. The assessment scale for global QOL consists of two questions and reveals only the underlying trends of HRQOL. As HRQOL is a multidimensional concept, its different domains, as measured with the EORTC questionnaires, must be considered when evaluating the effects of a disease and its treatment. There are intentions to develop a summary score that integrates the majority of functional and symptom scales, and to improve the evaluation of surveys of patient HRQOL (14); however, this approach may be limited by the multidimensional concept of QOL.

Increased global QOL over time in the malignant and GBM groups as observed during the present study may have been the result of various effects. It is conceivable that a positive effect of radiotherapy may increase HRQOL. An additional reason may be the response shift $(15,16)$ : The personal assessment of patients may change due to novel experiences, for instance if a patient recovered from a poor health status (including recovery 


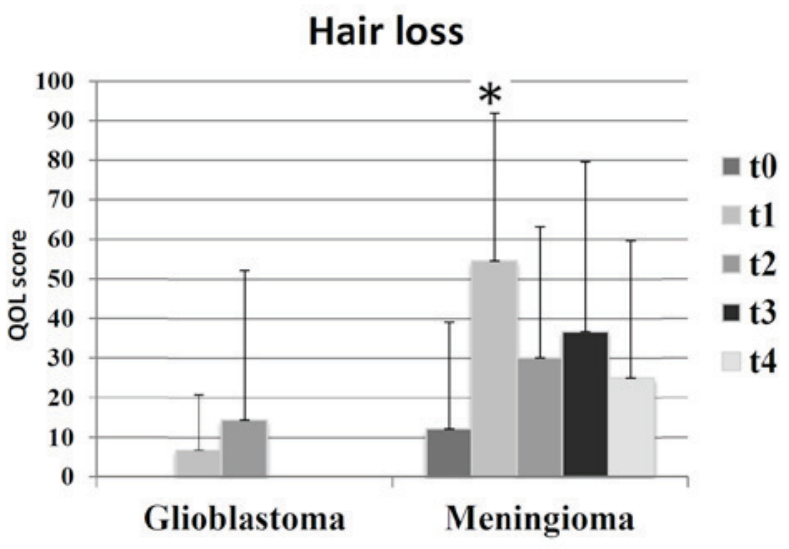

Figure 5. Symptom scales of alopecia in the meningioma and GBM groups at all times: $\mathrm{t} 0$ (prior to radiotherapy), $\mathrm{t} 1$ (subsequent to radiotherapy), $\mathrm{t} 2$ (after 3 months), t3 (after 6 months) and t 4 (after 12 months), as measured by the European Organization for Research and Treatment of Cancer BN20 questionnaire. A higher score indicated a worse quality of life. ${ }^{*} \mathrm{P}<0.01$. GBM, glioblastoma multiforme.

\section{Financial difficulties}

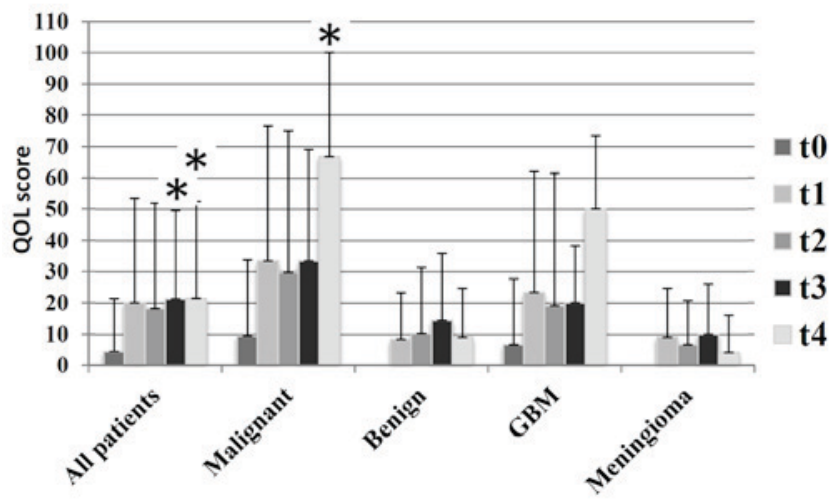

Figure 6. Symptom scales of financial difficulties in all groups and at all times: t0 (prior to radiotherapy), t1 (subsequent to radiotherapy), t2 (after 3 months), t 3 (after 6 months) and 4 (after 12 months), as measured by the European Organization for Research and Treatment of Cancer QLQ-C30 questionnaire. A higher score indicated a worse quality of life; ${ }^{*} \mathrm{P}<0.01$.

from treatment side effects), and so patients may score an improved HRQOL, in spite of deterioration of their condition. Also, patients with similar tumor types and objective health status may estimate their HRQOL in a different manner due to diverse expectations (17). It is also possible that the global QOL appears to increase due to selection effects. Patients with low HRQOL may only provide data at the beginning but not at later stages, due to mortality or non-response resulting from a worsening of their general health. Non-response may lead to sample distortion, and thus to an artificial improvement in the mean HRQOL.

It is unclear whether the decrease in functional scale in patients with malignant brain tumors is due to the cancer therapy or the tumor itself. A study of long-term survivors with low-grade gliomas suggested that the deterioration in QOL was not due to previous radiotherapy or chemotherapy, but due to tumor relapses (18). Therefore, the results of the present study demonstrate certain similarities to the previous studies, which may lead to the hypothesis that tumor relapses may be the predominant reason for decreasing functional scales during follow-up following radiotherapy.

During the study time frame, no patients succumbed to disease in the benign and meningioma groups, and the response rates were $\geq 68.8 \%$. Future uncertainty and headaches continuously decreased. The increase in QOL in the benign and meningioma groups appeared to be due to successful therapy. Functional scales of the QLQ-C30 remained stable during radiotherapy. Similar results were identified in a German study from 2013 (19), which evaluated 67 patients with meningioma during radiotherapy. In this prior study, the functional scales decreased shortly following radiotherapy and then normalized after 12 months. The study by Henzel et al (19) indicated that pain decreased subsequent to therapy, corresponding to the outcomes of the present study, which recorded decreasing headaches in patients following therapy.

At 11 (subsequent to radiotherapy) there were certain detectable adverse events. In particular, fatigue increased markedly $(\mathrm{t} 0=29 ; \mathrm{t} 1=54)$; this may be a direct acute effect of radiotherapy. Previous studies demonstrated that HRQOL decreased in patients with glioma who suffered from fatigue $(16,20)$. This is concordant with the observation of increased levels of fatigue in the GBM group ( $\mathrm{t} 0=37$ ), compared with in the meningioma group $(\mathrm{t} 0=23)$, prior to radiotherapy.

The objective adverse events, measured using CTCAE, were comparable with the subjective claims of the patients. Predominant subjective and objective areas of relevance were fatigue and alopecia. However, there was a distinct pattern of occurrence of symptoms. All patients with GBM objectively exhibited alopecia grade 1; however, only $73 \%$ of patients with meningioma experienced alopecia grade 1 , and $23 \%$ exhibited no alopecia. The meningioma group reported higher scales of alopecia on the QLQ-BN20 compared with the GBM group, which may be due to a differing standard of assessment for patients in evaluating their own HRQOL (16). Participants with a palliative diagnosis (GBM and malignant groups) considered alopecia to be a minor issue; conversely, participants with a curative diagnosis (meningioma and benign) considered alopecia a major issue. This result demonstrates the need for simultaneous analyses of objective and subjective adverse events in order to improve the HRQOL of patients. Quinten et al (7) identified that the simultaneous examination also assists in predicting survival. Physicians may have different assessment criteria for individual symptoms due to their practical experience with patients with oncology. Therefore, there is a risk that the effects of certain symptoms may not be recognized, which then leads to a negative effect on HRQOL.

One year following radiotherapy, participants with malignant brain tumors reported increased financial difficulties, particularly for $\mathrm{t} 4$, while the financial problems in the benign and meningioma groups were reversed. The reason may be that patients with benign brain tumors go back to work, while patients with malignant brain tumors have not.

A limitation of the present study was the low number of participants $(n=30)$. Data collection over longer time periods, possibly with a larger number of participants or a multi-centric study design, may produce more significant data. Due to the limited sample, there were no additional subdivisions of the groups (sex, age or total dose). In future studies, a subdivision may 
provide more comprehensive results. A previous study suggested that differences exist between male and female participants (21). Additional information concerning depression and anxiety may also assist in improving the HRQOL of patients. A previous study demonstrated that patients suffering from depression and anxiety exhibited poorer HRQOL (22). Poorer HRQOL and depression are also negative predictors of survival (23).

In conclusion, although there was no statistically significant improvement in global QOL, the present study demonstrated a moderately clinically relevant improvement to HRQOL. Sub-features of HRQOL, such as headaches and visual disturbances, may also be improved. It may be hypothesized that the simultaneous collection of objective and subjective therapeutic effects are important. Thus, the present study may assist in identifying indications, which may be valuable for patient counseling. Nevertheless, additional studies are necessary to improve the HRQOL of patients with brain tumors. There is a need for tolerable and effective therapies, intensive treatment of side effects and additional efforts to assist patients to continue their daily life.

\section{References}

1. Louis DN, Perry A, Reifenberger G, von Deimling A, Figarella-Branger D, Cavenee WK, Ohgaki H, Wiestler OD, Kleihues P and Ellison DW: The 2016 World Health Organization classification of tumors of the central nervous system: A summary. Acta Neuropathol 131: 803-820, 2016.

2. Velikova G, Coens C, Efficace F, Greimel E, Groenvold M, Johnson C, Singer S, van de Poll-Franse L, Young T and Bottomley A: Health-related quality of life in EORTC clinical trials-30 years of progress from methodological developments to making a real impact on oncology practice. EJC Suppl 10: 141-149, 2012.

3. Outcomes of cancer treatment for technology assessment and cancer treatment guidelines. American Society of Clinical Oncology. J Clin Oncol 14: 671-679, 1996.

4. Jocham HR, Dassen T, Widdershoven G and Halfens R: Quality of life in palliative care cancer patients: A literature review. J Clin Nurs 15: 1188-1195, 2006.

5. Steinmann D, Vordermark D, Geinitz H, Aschoff R, Bayerl A, Gerstein J, Hipp M, van Oorschot B, Wypior HJ and Schäfer C: Proxy assessment of patients before and after radiotherapy for brain metastases. Results of a prospective study using the DEGRO brain module. Strahlenther Onkol 189: 47-53, 2013.

6. Velikova G, Booth L, Smith AB, Brown PM, Lynch P, Brown JM and Selby PJ: Measuring quality of life in routine oncology practice improves communication and patient well-being: A randomized controlled trial. J Clin Oncol 22: 714-724, 2004.

7. Quinten C, Maringwa J, Gotay CC, Martinelli F, Coens C, Reeve BB, Flechtner H, Greimel E, King M, Osoba D, et al: Patient self-reports of symptoms and clinician ratings as predictors of overall cancer survival. J Natl Cancer Inst 103: 1851-1858, 2011.

8. Aaronson NK, Ahmedzai S, Bergman B, Bullinger M, Cull A, Duez NJ, Filiberti A, Flechtner H, Fleishman SB, de Haes JC, et al: The European organization for research and treatment of cancer QLQ-C30: A quality-of-life instrument for use in international clinical trials in oncology. J Natl Cancer Inst 85: 365-376, 1993.
9. Chow R, Lao N, Popovic M, Chow E, Cella D, Beaumont J, Lam H, Pulenzas N, Bedard G, Wong E, et al: Comparison of the EORTC QLQ-BN20 and the FACT-Br quality of life questionnaires for patients with primary brain cancers: A literature review. Support Care Cancer 22: 2593-2598, 2014.

10. Taphoorn MJ, Claassens L, Aaronson NK, Coens C, Mauer M, Osoba D, Stupp R, Mirimanoff RO, van den Bent MJ and Bottomley A; EORTC Quality of Life Group, and Brain Cancer, NCIC and Radiotherapy Groups: An international validation study of the EORTC brain cancer module (EORTC QLQ-BN20) for assessing health-related quality of life and symptoms in brain cancer patients. Eur J Cancer 46: 1033-1040, 2010.

11. Fayers PM, Aaronson NK, Bjordal K, Groenvold M, Curran D and Bottomley A; The EORTC Quality of Life Group: The EORTC QLQ-C30 Scoring Manual. 3rd edition. European Organisation for Research and Treatment of Cancer, Brussels, 2001.

12. Cancer Therapy Evaluation Program: Common Terminology Criteria for Adverse Events, Version 3.0. DCTD, NCI, NIH, DHHS, 2003. https://ctep.cancer.gov/protocoldevelopment/electronic_applications/docs/ctcaev3.pdf. Accessed August 9, 2006.

13. Osoba D, Rodrigues G, Myles J, Zee B and Pater J: Interpreting the significance of changes in health-related quality-of-life scores. J Clin Oncol 16: 139-144, 1998.

14. Giesinger JM, Kieffer JM, Fayers PM, Groenvold M, Petersen MA, Scott NW, Sprangers MA, Velikova G and Aaronson NK; EORTC Quality of Life Group: Replication and validation of higher order models demonstrated that a summary score for the EORTC QLQ-C30 is robust. J Clin Epidemiol 69: 79-88, 2016

15. Schumacher J, Klaiberg A and Brähler E: Diagnostische Verfahren zu Lebensqualität und Wohlbefinden. 1st edition. Göttingen, Hogrefe, Verlag für Psychologie, pp9-24, 2003.

16. Taphoorn MJ, Sizoo EM and Bottomley A: Review on quality of life issues in patients with primary brain tumors. Oncologist 15: 618-626, 2010.

17. Carr AJ, Gibson B and Robinson PG: Measuring quality of life: Is quality of life determined by expectations or experience? BMJ 322: 1240-1243, 2001.

18. Okita Y, Narita Y, Miyahara R, Miyakita Y,Ohno M and Shibui S: Health-related quality of life in long-term survivors with Grade II gliomas: The contribution of disease recurrence and Karnofsky Performance Status. Jpn J Clin Oncol 45: 906-913, 2015.

19. Henzel M, Fokas E, Sitter H, Wittig A and Engenhart-Cabillic R: Quality of life after stereotactic radiotherapy for meningioma: A prospective non-randomized study. J Neurooncol 113: 135-141, 2013.

20. Aprile I, Chiesa S, Padua L, Di Blasi C, Arezzo MF, Valentini V, DiStasio E and Balducci M: Occurrence and predictors of the fatigue in high-grade glioma patients. Neurol Sci 36: 1363-1369, 2015.

21. Sehlen S, Hollenhorst H, Schymura B, Herschbach P, Aydemir U, Firsching $M$ and Dühmke E: Psychosocial stress in cancer patients during and after radiotherapy. Strahlenther Onkol 179: 175-180, 2003.

22. Lucchiari C, Botturi A, Silvani A, Lamperti E, Gaviani P, Innocenti A, Finocchiaro CY, Masiero M and Pravettoni G: Cognitive strategies and quality of life of patients with high-grade glioma. Support Care Cancer 23: 3427-3435, 2015.

23. Mainio A, Tuunanen S, Hakko H, Niemelä A, Koivukangas J and Räsänen P: Decreased quality of life and depression as predictors for shorter survival among patients with low-grade gliomas: A follow-up from 1990 to 2003. Eur Arch Psychiatry Clin Neurosci 256: 516-521, 2006. 